\title{
Abundances in 4 Lacertae and $\nu$ Cephei
}

\author{
Kutluay Yüce \\ Ankara University, Faculty of Science, Department of Astronomy and Space Sciences, \\ TR-06100, Tandoğan, Ankara, Turkey \\ e-mail: kyuce@astro1.science.ankara.edu.tr
}

\begin{abstract}
Using Kurucz's ATLAS9 model atmospheres I performed LTE fine analyses of the supergiants $4 \mathrm{Lac}$ and $\nu$ Cep. The spectra were obtained at $1.22-\mathrm{m}$ telescope of the Dominion Astrophysical Observatory (DAO) by Saul J. Adelman. The atmospheric parameters were derived using the wings of $\mathrm{H} \beta$ and $\mathrm{H} \gamma$ profiles for each star, and from Fe I/II, Fe II/III, and $\mathrm{Si}$ II/III equilibria for $4 \mathrm{Lac}$, and from Fe I/II and $\mathrm{Cr}$ I/II equilibria for $\nu$ Cep. A microturbulence of $2.7 \mathrm{~km} \mathrm{~s}^{-1}$ for $4 \mathrm{Lac}$ was found from Fe II lines while a mean value of $5.2 \mathrm{~km} \mathrm{~s}^{-1}$ for $\nu$ Cep from Cr II, Ti II and Fe II lines. The rotational and macroturbulent velocities are, respectively, $14 \pm$ $2 \mathrm{~km} \mathrm{~s}^{-1}$ and $15 \pm 2 \mathrm{~km} \mathrm{~s}^{-1}$ for $4 \mathrm{Lac}$, and $26 \pm 2 \mathrm{~km} \mathrm{~s}^{-1}$ and $12 \pm 2 \mathrm{~km} \mathrm{~s}^{-1}$ for $\nu$ Cep. Their $\mathrm{He}, \mathrm{CNO}$ and light element abundances are solar or overabundant while their iron peak and heavy element abundances are solar or underabundant. The derived results show that 4 Lac has nuclearly processed matter in its photosphere while $\nu$ Cep does not.
\end{abstract}

Keywords. Stars; abundances, stars: evolution, stars: individual; (4 Lac, $\nu$ Cep)

\section{Introduction}

4 Lacertae (HD 212593, HR 8541, BD +48 3715, SAO 51970, HIP 110609) and $\nu$ Cephei (HD 207260, HR 8334, BD +60²288, SAO 19624, HIP 107418; $10 \mathrm{Cep)} \mathrm{were} \mathrm{classified} \mathrm{as}$ B9 Iab and A2 Ia, respectively, by Morgan \& Roman (1950), Slettebak (1954) and Stock (1956). $\nu$ Cephei is a member of Cep OB2 association (Humphreys 1978). Hill et al. (1986) indicate that $4 \mathrm{Lac}$ is a member of Lac OB1, but this is uncertain because of its large residual for the $\mathrm{Mv}-\mathrm{W}(\mathrm{H} \gamma)$ calibration (see also Humphreys 1978).

For $4 \mathrm{Lac}$ and $\nu$ Cep which are for the most part unstudied supergiants in literature, their optical spectrophotometry remains to be obtained. Verdugo et al. (1999) derived as the atmospheric parameters of $4 \mathrm{Lac}\left(T_{\mathrm{eff}}=10000 \mathrm{~K}, \log g=1.5\right)$ from Balmer fits using ATLAS9 LTE line blanketed plane parallel static model atmospheres (Kurucz 1993). The effective temperature values are $10300 \mathrm{~K}$ (Schmidt-Kaler 1982) and $9932 \mathrm{~K}$ (Underhill 1982) for $4 \mathrm{Lac}$, and $9080 \mathrm{~K}$ (Schmidt-Kaler 1982) and $9120 \mathrm{~K}$ (Garmany \& Stencel 1992) for $\nu$ Cep.

\section{Spectra}

Saul J. Adelman obtained $2.4 \AA_{\mathrm{mm}^{-1}}$ DAO CCD spectrograms which cover almost $63 \AA$ for the SITe 2 and $147 \AA$ for the SITe 4 . Spectra between 3830 and $4950 \AA$ have $S / N>250$. The combination of these electronic devices with a high resolution spectrograph enables one to analyze profiles of even faint lines with high accuracy. I used the computer graphs program REDUCE and VLINE (Hill \& Fisher 1986) to normalize and measure the spectra. The scattered light in the direction of the dispersion was corrected as part of the extraction program CCDSPEC (Gulliver \& Hill 2002).

My average radial velocity was $-21.9 \pm 5.1 \mathrm{~km} \mathrm{~s}^{-1}$ with an amplitude of $13.3 \mathrm{~km} \mathrm{~s}^{-1}$ 


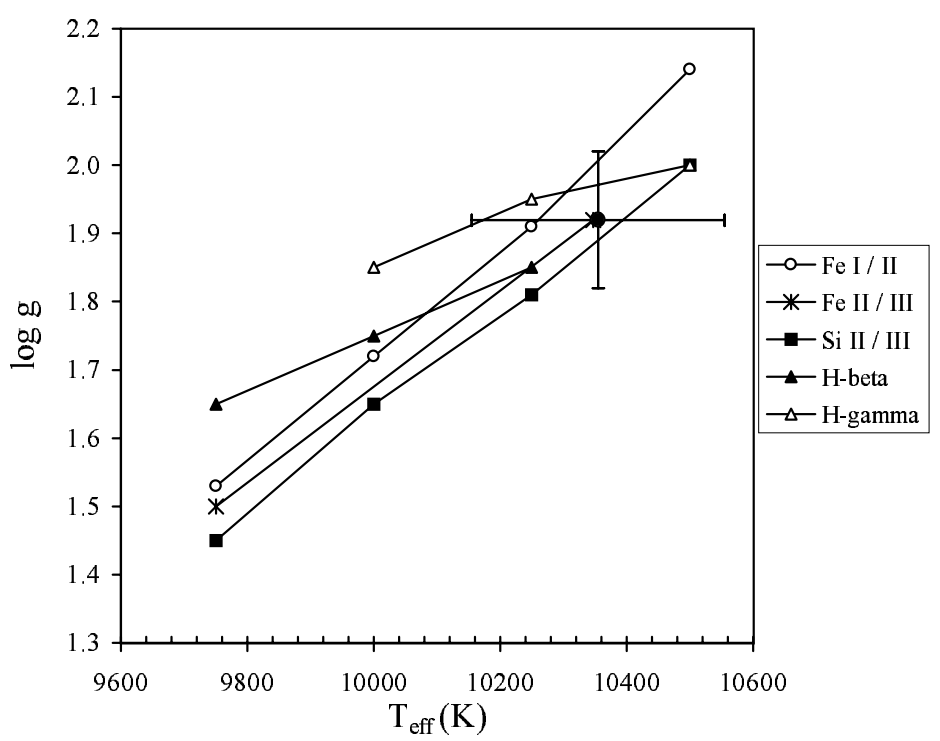

Figure 1. $T_{\text {eff }}-\log g$, a Kiel diagram of 4 Lac.

for $4 \mathrm{Lac}$, and $-20.1 \pm 5.0 \mathrm{~km} \mathrm{~s}^{-1}$ with an amplitude of $14.8 \mathrm{~km} \mathrm{~s}^{-1}$ for $\nu$ Cep. Values from literature are between -28 and $-20 \mathrm{~km} \mathrm{~s}^{-1}$ for $4 \mathrm{Lac}$ whereas they are between -25 and $-15 \mathrm{~km} \mathrm{~s}^{-1}$ for $\nu$ Cep.

\section{Determinations}

\subsection{Atmospheric parameters}

The effective temperature and surface gravity were derived using the Kiel diagram for each star. They contain pairs from ionization equilibria and the observed Balmer line wing fits with the calculated SYNTHE (Kurucz \& Avrett 1981) profiles (Figures 1 and 2 ). The best atmospheric parameter pairs are $T_{\text {eff }}=10350 \mathrm{~K}$ and $\log g=1.92$ for $4 \mathrm{Lac}$, $T_{\text {eff }}=8500 \mathrm{~K}$ and $\log g=1.25$ for $\nu$ Cep. The adopted atmosphere parameters are marked with closed circles in Figures 1 and 2.

Lemke (1989) finds that for main sequence stars the mean accuracy of atmospheric parameters are $\pm 200 \mathrm{~K}$ in effective temperature and probably not larger than \pm 0.2 dex in surface gravity. These values are in accord with uncertainties of seven A0-A2 type supergiants derived from $\mathrm{H} \gamma$ fits and $\mathrm{Mg}$ I/II ionization equilibrium by Venn (1995a). For the Kiel diagram of $\nu$ Cep, three criteria have lines parallel to one another and thus it is difficult to get good range estimates. An examination of the Kiel diagram of $4 \mathrm{Lac}$ by considering the intersections of the criteria indicates a maximum range of $400 \mathrm{~K}$ in effective temperature and 0.2 dex in surface gravity. Thus, the uncertainities found by Lemke appear also to be appropriate for 4 Lac and $\nu$ Cep.

\subsection{Microturbulence and $\mathrm{He} / \mathrm{H}$ ratio}

The microturbulent velocity was determined from Fe II lines in 4 Lac, and from Cr II, Ti II, and Fe II lines in $\nu$ Cep. I used species having at least 20 lines with equivalent widths $5 \mathrm{~m} \AA$ or more including 5 with equivalent widths between 90 and $150 \mathrm{~m} \AA$. In addition, the lines were selected as they are unblended and have reliable $g f$ values. Their abundances were calculated with WIDTH9 (Kurucz 1993) assuming various microturbulent velocities for a range of possible values. The adopted values for each species resulted in no dependence of 


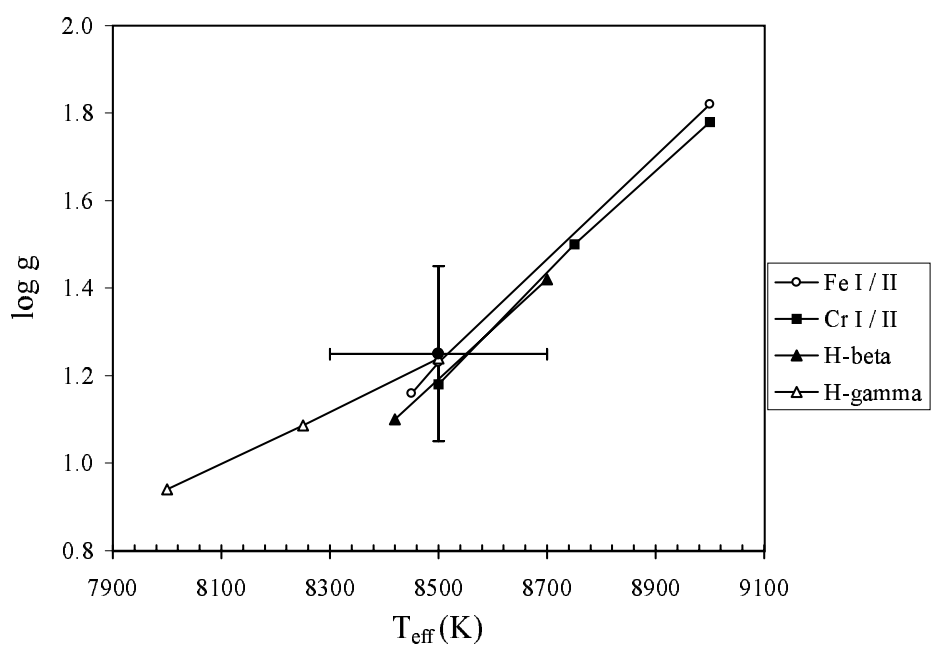

Figure 2. $T_{\text {eff }}-\log g$, a Kiel diagram of $\nu$ Cep.

the derived abundances on the equivalent width. Thus, the results tell us that 4 Lac has the microturbulent velocity of $2.7 \mathrm{~km} \mathrm{~s}^{-1}$ whereas $\nu$ Cep has the mean value of $5.2 \mathrm{~km} \mathrm{~s}^{-1}$.

The $\mathrm{He} / \mathrm{H}$ ratio were derived comparing the observed $\mathrm{He}$ I line profiles with synthesized spectra using the program SYNSPEC (Hubeny et al. 1994). The mean of derived He/H values from nine lines of $4 \mathrm{Lac}$ is $0.154 \pm 0.019$ whereas it is $0.136 \pm 0.004$ for $\nu$ Cep.

\subsection{Other elemental abundances}

Using the derived microturbulent velocities $\xi=2 \mathrm{~km} \mathrm{~s}^{-1}$ for $4 \mathrm{Lac}$ and $4 \mathrm{~km} \mathrm{~s}^{-1}$ for $\nu$ Cep, selected atomic parameters, and ATLAS9 model atmospheres $\log \left(N / N_{\mathrm{T}}\right)$ values were found for each line with a well determined equivalent width using WIDTH9. All abundance results, their comparisons with the Sun and similar type supergiants are presented in Yüce (2004) along with estimates of the sensitivities of the abundances on the atmospheric parameter errors using two models one of whose effective temperature is $200 \mathrm{~K}$ hotter and the other of which has $\log g 0.2$ dex greater than the adopted values.

The mean abundances show that $4 \mathrm{Lac}$ is a slightly metal richer supergiant than $\nu$ Cep in light elements. On the other hand, $4 \mathrm{Lac}$ is more metal poor than $\nu$ Cep for the iron peak elements. Most of $\nu$ Cep's abundances are close to solar with a few exceptions: Ca, $\mathrm{Sc}, \mathrm{Ti}$, and $\mathrm{Y}$ while the earlier type $4 \mathrm{Lac}$ is more variable in $\mathrm{N}, \mathrm{Si}, \mathrm{Ar}, \mathrm{Ca}, \mathrm{Ti}, \mathrm{Cr}$, and Sr. Nevertheless, both these early type supergiants tend to show the same abundance patterns relative to the solar, namely both stars seem to show that He, CNO and light element (Mg, Al, Si, S, Ar, Ca) abundances have solar values or are overabundant while the iron group elements ( $\mathrm{Sc}, \mathrm{Ti}, \mathrm{V}, \mathrm{Cr}, \mathrm{Mn}, \mathrm{Fe}, \mathrm{Ni}$ ) and the heavy elements (Sr, Y, $\mathrm{Zr}$ ) abundances are generally solar or underabundant. Si and $\mathrm{Ca}$, which are prototypical $\alpha$-elements synthesized during hydrostatic burning of massive stars (Woosley \& Weaver 1995), are overabundant. Sr and Zr from s-process elements are mildly underabundant in $4 \mathrm{Lac}$ and $\nu \mathrm{Cep}$, respectively.

\subsection{Macroturbulent and rotational velocities}

In this study, first estimates of the rotational velocities for $4 \mathrm{Lac}$ and $\nu$ Cep were determined from measuring unblended symmetric lines (for examples; Fe II, Ti II) using VLINE (Hill \& Fisher 1986). They are $16 \mathrm{~km} \mathrm{~s}^{-1}$ and $21 \mathrm{~km} \mathrm{~s}^{-1}$, respectively.

To determine the rotational $(v \sin i)$ and the macroturbulent $(\zeta)$ velocities for 4 Lac and 


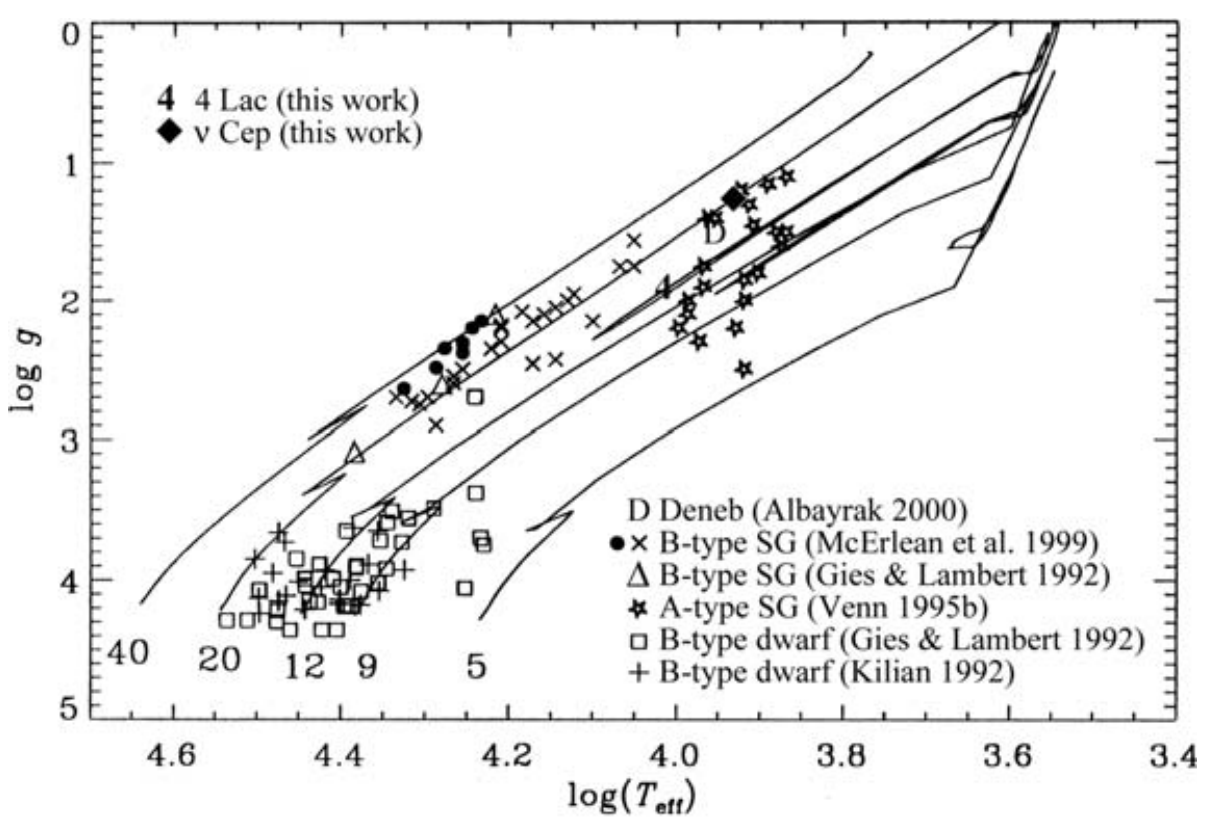

Figure 3. Positions of $4 \mathrm{Lac}, \nu$ Cep and some stars in $T_{\text {eff }}-\log g$ plane.

$\nu$ Cep, the program SYNTHE (Kurucz \& Avrett 1981) was used to calculate a synthetic spectrum for $\lambda \lambda 4480-4559$, a region with clean strong lines for each star. The synthetic spectra were compared to the normalized observations. Then the values of macroturbulent and rotational velocities were changed until the best fits were reached. For $4 \mathrm{Lac}$, the best values are $v \sin i=14 \pm 2 \mathrm{~km} \mathrm{~s}^{-1}$ and $\zeta=15 \pm 2 \mathrm{~km} \mathrm{~s}^{-1}$ while the optimum values for $\nu$ Cep are $v \sin i=26 \pm 2 \mathrm{~km} \mathrm{~s}^{-1}$ and $\zeta=12 \pm 2 \mathrm{~km} \mathrm{~s}^{-1}$.

\subsection{Evolutionary status of 4 Lac and $\nu$ Cep}

The chemical abundances of $4 \mathrm{Lac}$ and $\nu$ Cep with $M>10 M_{\odot}$ help to illuminate the evolution of massive stars. The CNO surface abundances of a supergiant are the Zero Age Main Sequence (ZAMS) surface values that have possibly been modified by mixing (dredge-up) between the interior and outer envelope of a star. Since CNO elements only act as catalysts during hydrogen burning for a massive main sequence star, that a reduction of the abundances of $\mathrm{C}$ and of $\mathrm{O}$ (only slightly) and the increase of the abundance of $\mathrm{N}$ with the sum of the nuclei remaining constant is predicted. For 4 Lac, the surface abundances ( $\mathrm{C}$ solar, $\mathrm{N}$ overabundant, and $\mathrm{O}$ slightly underabundant) are evidence for $\mathrm{CNO}$ core-processed material being present in the photosphere. The atmospheric abundances of $\nu \mathrm{Cep}$ (near solar $\mathrm{C}$ and $\mathrm{N}$ and a slight underabundance of $\mathrm{O}$ ) are in agreement with the model in which the star initiated helium core ignition without visiting the red giant branch (RGB). The evolutionary path of $\nu$ Cep coincides with the non-rotating model given for the track of a $20 M_{\odot}$ star by Maeder \& Meynet (2001).

I also compared the atmospheric parameters the supergiants with the theoretical calculations. Figure 3 shows the locations of our my supergiants, along with the solar metallicity evolutionary tracks of Schaller et al. (1992). It also contains similar type stars. This study's results put $4 \mathrm{Lac}$ on the $12 \mathrm{M}_{\odot}$ track in the region near Venn's supergiants. This agrees with the mass values of $4 \mathrm{Lac}$ in literature $\left(10-11 M_{\odot}\right)$. According to the Figure, a main sequence early B star was the progenitor of 4 Lac. The atmospheric parameters of $\nu$ Cep place it on the $20 M_{\odot}$ path which is a little above the track given by Lamers et al. 


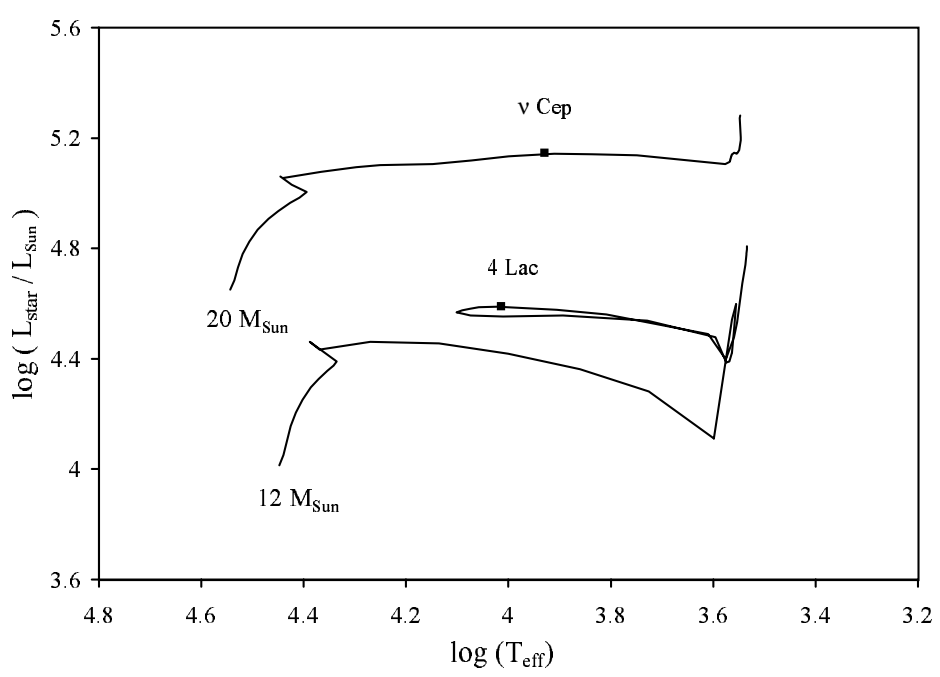

Figure 4. Positions of $4 \mathrm{Lac}$ and $\nu$ Cep on the theoretical evolutionary tracks of Schaller et al. (1992).

(1995, $\left.16 M_{\odot}\right)$. Thus $\nu$ Cep is more luminous than many of Venn's stars and in agreement with the moderate/normal processed B-type supergiants. $\nu$ Cep's main sequence progenitor would be of O-type.

Thus when I examined the positions of two supergiants on the $\left(\log L-\log T_{\text {eff }}\right)$ theoretical evolutionary paths of Schaller et al. (1992);

(i) The values of $R=60_{\odot}$ (Leitherer 1988) and $T_{\text {eff }}=10350 \mathrm{~K}$ (this study) of $4 \mathrm{Lac}$ put it on the $12 M_{\odot}$ evolutionary path (Figure 4 ). The star has evolved back from the red supergiant phase (on a blue loop). This position is the same as that in Figure 3 from its atmospheric parameters. Schaller et al. (1992) give the age of this point as 16 million years. It seems to be confirmed with the non-rotating model of $12 M_{\odot}$ by Meynet \& Maeder (2003). This age is in agreement with that of LAC OB1 association (16-25 x10 6 year, Blaauw 1958). This increases the probability that $4 \mathrm{Lac}$ is a member of LAC OB1.

(ii) $\nu$ Cep is on the $20 M_{\odot}$ evolution calculation (Figure 4 ) which provides an age of $8 \times 10^{6}$ year for that point, which was confirmed by Meynet \& Maeder (2003). This age agrees with CEP OB2 (7-3 x $10^{6}$ year in its a and b sub-associations, Simonson \& Someren Greve (1976)).

(iii) Both 4 Lac and $\nu$ Cep are metal poor compared with the Sun. This study shows 4 Lac is slightly more metal poor than $\nu$ Cep, and 4 Lac has a bluer loop in HRD.

\section{Acknowledgments}

I thank my primary supervisor Dr. Saul J. Adelman and Drs. Cemal Aydin and Berahitdin Albayrak. I also thank Dr. James E. Hesser, Director, Dominion Astrophysical Observatory, Herzberg Institute of Astrophysics for the observing time used by Dr. Adelman to obtain the spectra I used for this study. 


\section{References}

Albayrak B., 2000, A\& $A, 364,237$

Blaauw A., 1958, AJ, 63, 186

Garmany C. D., Stencel R. E., 1992, A\&AS, 94, 211

Gies D. R., Lambert D. L., 1992, ApJ, 387, 673

Gulliver A. F., Hill G., 2002, Astronomical Data Analysis Software and Systems XI, ASP Conference Proceedings, Vol. 281, p. 351

Hill G., Fisher W. A., 1986, Publ. Dominion Astrophys. Obs. Victoria, 16, No. 13

Hill G. M., Walker G. A. H., Yang S., 1986, PASP, 98, 1186

Hubeny I., Lanz T., Jeffery C. S., 1994, Daresbury Lab. New. Anal. Astron. Spectra No. 20, p. 30

Humphreys R. M., 1978, ApJS, 38, 309

Kilian J., 1992, A\&\&A, 262, 171

Kurucz R. L., 1993, ATLAS9 Stellar Atmosphere Programs and $2 \mathrm{~km} / \mathrm{s}$ grid, Kurucz CM-ROM No. 13, Smithsonian Astrophysical Observatory, Cambridge, MA

Kurucz R. L., Avrett E. H., 1981, Smithsonian Astrophys. Obs. Spec. Rep., 391

Lamers H. J. G. L. M., Snow T. P., Lindholm D. M., 1995, ApJ, 455, 269

Leitherer C., 1988, ApJ, 326, 356

Lemke M., 1989, $A \& A$, 225, 125

Maeder A., Meynet G., 2001, A\&A, 373, 555

McErlean N. D., Lennon D. J., Dufton P. L., 1999, A\&A, 349, 553

Meynet G., Maeder A., 2003, A\&\&A, 404, 975

Morgan W. W., Roman N. G., 1950, ApJ, 112, 362

Schaller G., Schaerer D., Meynet G., Maeder A., 1992, A\&AS, 96, 269

Schmidt-Kaler Th., 1982, Landort-Börnstein, Vol.2, Subvol. b, p.455, Springer Verlag

Simonson S. C., van Someren Greve H. W., 1976, A\& A, 49, 343

Slettebak A., 1954, ApJ, 119, 146

Stock J., 1956, ApJ, 123, 253

Underhill A. B., 1982, B stars with and without emission lines (Part I). NASA SP-456, eds. A. B. Underhill, V. Doazan, (NASA Washington DC)

Venn K. A., 1995a, ApJS, 99, 659

Venn K. A., 1995b, ApJ, 449, 839

Verdugo E., Talavera A., Gomez de Castro A. I., 1999, A\&SA 346, 819

Woosley S. E., Weaver T. A., 1995, ApJS, 101, 181

Yüce K., 2004, Baltic Astronomy, in press 